\title{
Sending-or-not-sending twin-field quantum key distribution with discrete-phase-randomized weak coherent states
}

\author{
Cong Jiang, ${ }^{1,2}$ Zong-Wen $\mathrm{Yu}^{2,3}$ Xiao-Long $\mathrm{Hu}^{2}{ }^{2}$ and Xiang-Bin Wang $\oplus^{1,2,4,5, *}$ \\ ${ }^{1}$ Jinan Institute of Quantum Technology, Jinan, Shandong 250101, People's Republic of China \\ ${ }^{2}$ State Key Laboratory of Low-Dimensional Quantum Physics, Department of Physics, Tsinghua University, \\ Beijing 100084, People's Republic of China \\ ${ }^{3}$ Data Communication Science and Technology Research Institute, Beijing 100191, People's Republic of China \\ ${ }^{4}$ Shanghai Branch, CAS Center for Excellence and Synergetic Innovation Center in Quantum Information and Quantum Physics, \\ University of Science and Technology of China, Shanghai 201315, People's Republic of China \\ ${ }^{5}$ Shenzhen Institute for Quantum Science and Engineering and Physics Department, Southern University of Science and Technology, \\ Shenzhen 518055, China
}

(Received 26 August 2020; accepted 14 October 2020; published 1 December 2020)

\begin{abstract}
In many security proofs of quantum key distribution, the random phases of coherent states are assumed to be continuously modulated. However, in practice, we can only take discrete phase randomization to coherent-state sources. In this paper, we study the sending-or-not-sending (SNS) protocol with discrete-phase-randomized coherent states. We present the security proof of the SNS protocol with discrete phase modulation. We then present analytic formulas for key rate calculation. With the decoy-state method and the properties of trace distance, we get the analytical formula of the upper bound of the phase-flip error rate. We also get the lower bound of the yield of untagged bits, which can be calculated by either analytical formula or linear programming. Our numerical simulation results show that with only six phase values, the key rates of the SNS protocol can exceed the linear bound and, with 12 phase values, the key rates are very close to the results of the SNS protocol with continuously modulated phase randomization.
\end{abstract}

DOI: 10.1103/PhysRevResearch.2.043304

\section{INTRODUCTION}

The theories and experiments of quantum key distribution (QKD) [1-24] have been widely studied since the first QKD protocol was proposed by Bennett and Brassard in 1984 (BB84) [1]. The secure key rate and the distance are central issues in practical application of QKD. In particular, the decoy-state method [10-12] improves the relationship between key rate $R$ and channel transmittance $\eta$ from square scale, $R \sim O\left(\eta^{2}\right)$, to linear scale, $R \sim O(\eta)$. Recently, the idea of twin-field QKD (TFQKD) [25] and its variants [26-40] has further improved the key rate to the scale of the square root of channel transmittance, $R \sim O(\sqrt{\eta})$. So far, the TFQKD has been demonstrated by a number of experiments [20,41-44].

Among all the variants of TFQKD, the sending-or-notsending (SNS) protocol [26] together with its modified protocols $[35,38,45]$ has attracted much attention due to its large noise tolerance and high key rate. Moreover, the SNS

\footnotetext{
*Corresponding author: xbwang@mail.tsinghua.edu.cn; also at Center for Atomic and Molecular Nanosciences, Tsinghua University, Beijing 100084, People's Republic of China.

Published by the American Physical Society under the terms of the Creative Commons Attribution 4.0 International license. Further distribution of this work must maintain attribution to the author(s) and the published article's title, journal citation, and DOI.
}

protocol has the unique advantage that the traditional decoystate method directly applies, which makes the finite-key analysis very efficient. The SNS protocol has been experimentally demonstrated in a proof of principle in Ref. [41] and realized in real optical fiber with the finite-key effects taken into consideration [20,42]. Notably, the SNS protocol has been experimentally demonstrated over $509 \mathrm{~km}$ of optical fiber [20], which is the longest secure distance of QKD in optical fiber.

In practice, we need the decoy-state method [10-12] to assure the security of those protocols with imperfect sources. In the traditional decoy-state method, phase randomization is requested so that the source state can be regarded as the classical mixture of different photon-number states. However, perfect phase randomization by continuous modulation is technically not likely. In a real experiment, the phases of weak-coherent-state (WCS) sources are discretely modulated to $\frac{2 m \pi}{N}$, where $m=0,1,2, \ldots, N-1$ and $N$ is always an even number. The major difference between the discrete modulation and continuous modulation is that the actual states of the latter case are diagonal in the Fock basis while those of the former case are not.

To close the gap between the theory and experiment, we study the SNS protocol with discrete-phase-randomized WCS sources. Although the effects of discrete phase randomization have been studied for protocols such as the traditional decoy-state BB84 [10-12] in Ref. [46], the measurementdevice-independent QKD (MDIQKD) [15,16,47-52] in 
Refs. [53,54], and the nonpostselection protocol [29,30] of TFQKD in Ref. [55], no investigation has been done on the SNS protocol. Here, we study this based on the structure of the SNS protocol. We prove its security and present the formulas for key rate calculation. Thanks to the tagged model proposed in Ref. [56], we can get the secure key rate of a QKD protocol with imperfect sources. The key idea is that we can divide the sifted keys in the signal windows into two classes: the untagged bits and the tagged bits, where the maximum information leakage of the untagged bits can be measured by their corresponding phase-flip error rate and the bit values of the tagged bits are assumed to be known to the eavesdropper. Unlike other protocols with discrete-phase-randomized WCS sources [46,53-55,57], we analytically get the formulas of the upper bound of the phase-flip error rate and the lower bound of the yield of untagged bits while the others have to solve linear programming problems. Our numerical simulation results show that with only six phase values, the key rates of the SNS protocol can exceed the PLOB bound, the linear bound of the key rate established by Pirandola, Laurenza, Ottaviani, and Banchi [24]. With 12 phase slices, the key rates are very close to the SNS protocol with continuously modulated phase-randomized WCS sources. As the property of no bit-flip error in the untagged bits still holds, we can directly apply the method of active-odd-parity pairing (AOPP) proposed in our previous work [35] to improve the key rate. The numerical results show that the advantage of the AOPP method still holds.

This paper is arranged as follows. We first introduce how to perform the SNS protocol with discrete-phase-randomized WCS sources. Then, based on the equivalent entanglement protocol of the SNS protocol, we show how to get the formula of the phase-flip error rate. We next show how to apply the decoy-state method to get the upper bound of the phase-flip error rate and the lower bound of the yield of untagged bits. With those bounds, we show some numerical results. The paper ends with concluding remarks.

\section{THE SNS PROTOCOL WITH DISCRETE-PHASE- RANDOMIZED WEAK-COHERENT-STATE SOURCES}

\section{A. The protocol}

The implementation process of the SNS protocol with discrete-phase-randomized WCS sources is similar to that of the original SNS protocol [26]. Here, we introduce the 4-intensity protocol as follows.

For each time window, Alice (Bob) randomly decides whether it is a decoy window or a signal window. If it is a decoy window, Alice (Bob) randomly chooses to prepare a pulse of state $|0\rangle,\left|e^{2 m \pi i / N} \sqrt{\mu_{x}}\right\rangle$ or $\left|e^{2 m^{\prime} \pi i / N} \sqrt{\mu_{y}}\right\rangle(|0\rangle$, $\left|e^{2 n \pi i / N} \sqrt{\mu_{x}}\right\rangle$ or $\left.\left|e^{2 n^{\prime} \pi i / N} \sqrt{\mu_{y}}\right\rangle\right)$, where $m, m^{\prime}, n, n^{\prime}$ are randomly chosen from $\{0,1,2, \ldots, N-1\}$ and $N$ is assumed to be an even number. If it is a signal window, Alice (Bob) randomly chooses to prepare a vacuum pulse or a pulse of state $\left|e^{2 l \pi i / N} \sqrt{\mu_{z}}\right\rangle$ with probabilities $1-\epsilon$ and $\epsilon$, respectively, where $l$ is randomly chosen from $\{0,1,2, \ldots, N-1\}$. If Alice (Bob) decides to send out a vacuum pulse (that is to say, sending nothing or not sending), Alice (Bob) takes the corresponding classical bit value as $0(1)$. If Alice (Bob) decides to send out a pulse of state $\left|e^{2 l \pi i / N} \sqrt{\mu_{z}}\right\rangle$, Alice (Bob) takes the corresponding classical bit value as $1(0)$.

Then Alice and Bob send their pulses to Charlie; Charlie is assumed to perform interferometric measurements on the received pulses and announce the measuring results to Alice and Bob. If only one of the two detectors clicks, Charlie would announce that this pulse pair causes a click and whether the left detector or right detector clicks. Alice and Bob take it as a one-detector heralded event. In the $Z$ basis, only those onedetector heralding events are regarded as effective events that contribute to the process of final key extraction, and the events where Charlie has two clicks or no clicks would be ignored.

Note that this sending by a small probability $\epsilon$ or not sending by probability $1-\epsilon$ is the heart of the SNS protocol [26]. Let us consider the ideal case that Charlie has perfect detectors and the channel transmittance $\eta$ between Alice and Bob is small. Then only for the event that at least one of Alice and Bob decides to send, it may cause a one-detector heralding event. The probability $\epsilon^{2}$ that both Alice and Bob decide to send is quite small. If Charlie's detectors are not perfect, there is another type of bit-flip error, the one-detector heralding event with both sides having decided not to send, whose probability is limited by the dark count rate of the detectors, which should be very small over a large range of distance. As was shown in Ref. [35], we can use larger values for the probability of the sending decision if we take error rejection with two-way classical communication in the data postprocessing.

After Alice and Bob repeat the above process many times, they acquire a series of data which are used to perform the data postprocessing.

The first step of data postprocessing is sifting. Alice and Bob first announce the type of each time window they decide. For a window that both Alice and Bob decide is a signal window, it is a $Z$ window. The corresponding bits of the one-detector heralded event of the $Z$ windows, which are also called the sifted keys, are used to extract the final keys. Except for the $Z$ windows, Alice and Bob announce the intensities and phases they decide in each window. For a window that both Alice and Bob decide is a decoy window, if the intensity of the pulse is $\mu_{x}$ and their phases satisfy

$$
1-\left|\cos \left(\frac{2 m \pi}{N}-\frac{2 n \pi}{N}\right)\right| \leqslant \delta,
$$

where $\delta$ is a positive number close to 0 , it is a $X_{1}$ window. The data of $X_{1}$ windows are used to estimate the phase-flip error rate of untagged bits. The data of other windows are used to perform the decoy-state method.

As the phases of WCS pulses in the $Z$ windows are never announced in the public channel, the density matrix of those WCS pulses is

$$
\rho_{z}=\frac{1}{N} \sum_{l=0}^{N-1}\left|e^{2 l \pi i / N} \sqrt{\mu_{z}}\right\rangle\left\langle e^{-2 l \pi i / N} \sqrt{\mu_{z}}\right| .
$$

For convenience, we define the approximated $j$-photon state $\left|\lambda_{j}\right\rangle$ in the following form [46]:

$$
\left|\lambda_{j}\right\rangle=\frac{1}{\sqrt{P_{j}\left(\mu_{z}\right)}} \sum_{k=0}^{\infty} \frac{\left(\sqrt{\mu_{z}}\right)^{k N+j}}{\sqrt{(k N+j) !}}|k N+j\rangle,
$$


and $j=0,1,2, \ldots, N-1$. It is easy to see that $\rho_{z}$ is a classical mixture of different $\left|\lambda_{j}\right\rangle$. Explicitly, we have

$$
\rho_{z}=\sum_{j=0}^{N-1} P_{j}\left(\mu_{z}\right)\left|\lambda_{j}\right\rangle\left\langle\lambda_{j}\right|,
$$

where

$$
P_{j}\left(\mu_{z}\right)=\sum_{k=0}^{\infty} \frac{\mu_{z}^{k N+j} e^{-\mu_{z}}}{(k N+j) !} .
$$

For the pulse pairs in the $X_{1}$ windows, we can select the pulse pairs whose phases satisfy $n \equiv(m+q) \bmod N$, where $q$ is a constant integer in this phase postselection process; the density matrix of those selected pulse pairs is

$$
\begin{aligned}
\rho_{X 1}(q)= & \frac{1}{N} \sum_{m=0}^{N-1}\left|e^{2 m \pi i / N} \sqrt{\mu_{x}}\right\rangle_{A}\left|e^{2 n \pi i / N} \sqrt{\mu_{x}}\right\rangle_{B} \\
& \times\left\langlee ^ { - 2 m \pi i / N } \sqrt { \mu _ { x } } | _ { A } \left\langle\left. e^{-2 n \pi i / N} \sqrt{\mu_{x}}\right|_{B}\right.\right.
\end{aligned}
$$

where the subscript $A$ and $B$ indicate Alice and Bob, respectively. After a simple calculation, one can find that $\rho_{X 1}(q)$ is actually the classical mixture of the state $\left|\varphi_{j}^{q}\right\rangle$, which is

$$
\rho_{X 1}(q)=\sum_{j=0}^{N-1} P X_{j}\left(\mu_{x}\right)\left|\varphi_{j}^{q}\right\rangle\left\langle\varphi_{j}^{q}\right|,
$$

where

$$
\begin{aligned}
\left|\varphi_{j}^{q}\right\rangle= & \frac{e^{-\mu_{x}}}{\sqrt{P X_{j}\left(\mu_{x}\right)}} \sum_{k=0}^{\infty} \sum_{k_{1}=0}^{k N+j} \frac{\left(\sqrt{\mu_{x}}\right)^{k N+j}}{\sqrt{k_{1} !\left(k N+j-k_{1}\right) !}} \\
& \times e^{\frac{2 \pi i}{N} q\left(k N+j-k_{1}\right)}\left|k_{1} ; k N+j-k_{1}\right\rangle,
\end{aligned}
$$

and the probability to obtain the state $\left|\varphi_{j}^{q}\right\rangle$ is

$$
\begin{aligned}
P X_{j}\left(\mu_{x}\right) & =\sum_{k=0}^{\infty} \sum_{k_{1}=0}^{k N+j} \frac{\mu_{x}^{k N+j} e^{-2 \mu_{x}}}{k_{1} !\left(k N+j-k_{1}\right) !} \\
& =\sum_{k=0}^{\infty} \frac{\left(2 \mu_{x}\right)^{k N+j} e^{-2 \mu_{x}}}{(k N+j) !}=P_{j}\left(2 \mu_{x}\right) .
\end{aligned}
$$

\section{B. The security analysis}

Following the security proof in Ref. [26], let us first consider the equivalent entanglement protocol of the SNS protocol.
In the entanglement protocol, for each time window, Alice and Bob preshare the entanglement state

$$
\begin{aligned}
\left|\Psi_{1}\right\rangle= & \frac{1}{\sqrt{2}}\left(\left|0 \lambda_{1}\right\rangle \otimes|\tilde{0} \tilde{0}\rangle+\left|\lambda_{1} 0\right\rangle \otimes|\tilde{1} \tilde{1}\rangle\right) \\
= & \frac{1}{\sqrt{2}}\left[\frac{1}{\sqrt{2}}\left(\left|0 \lambda_{1}\right\rangle+\left|\lambda_{1} 0\right\rangle\right) \otimes \frac{1}{\sqrt{2}}(|\tilde{0} \tilde{0}\rangle+|\tilde{1} \tilde{1}\rangle)\right. \\
& \left.+\frac{1}{\sqrt{2}}\left(\left|0 \lambda_{1}\right\rangle-\left|\lambda_{1} 0\right\rangle\right) \otimes \frac{1}{\sqrt{2}}(|\tilde{0} \tilde{0}\rangle-|\tilde{1} \tilde{1}\rangle)\right],
\end{aligned}
$$

where $|\tilde{0} \tilde{0}\rangle$ and $|\tilde{1} \tilde{1}\rangle$ are local states that only exist in Alice's and Bob's laboratories and $\left|0 \lambda_{1}\right\rangle$ and $\left|\lambda_{1} 0\right\rangle$ are real states that are sent to Charlie. According to the measurement results announced by Charlie, Alice and Bob can get a series of almost perfect entanglement state by applying entanglement purification to the local states. Two parameters are needed in the entanglement purification: The first is the bit-flip error rate in the $Z$ basis, $e_{z}$, and the second is the bit-flip error rate in the $X$ basis, $e^{\mathrm{ph}}$, which is also the phase-flip error rate in the $Z$ basis. Here, the $Z$ basis means $\{|\tilde{0}\rangle,|\tilde{1}\rangle\}$, and the $X$ basis means $\left\{\frac{1}{\sqrt{2}}(|\tilde{0}\rangle+|\tilde{1}\rangle), \frac{1}{\sqrt{2}}(|\tilde{0}\rangle-|\tilde{1}\rangle)\right\}$. Finally, by measuring the local states in the $Z$ basis, Alice and Bob can get secure final keys.

As Alice and Bob are only concerned about the secure final keys, they need not measure their local states after Charlie announces his measurement results, but they can just measure their local states before they send the real states to Charlie. If Alice and Bob each measure their local qubits in the $Z$ basis, it is equivalent to Alice and Bob randomly sending a pulse of state $\left|0 \lambda_{1}\right\rangle$ or $\left|\lambda_{1} 0\right\rangle$ to Charlie. If Alice and Bob each measure their local qubits in the $X$ basis, it is equivalent to Alice and Bob randomly sending a pulse of state $\left|\chi_{0}\right\rangle=$ $\frac{1}{\sqrt{2}}\left(\left|0 \lambda_{1}\right\rangle+\left|\lambda_{1} 0\right\rangle\right)$ or $\left|\chi_{1}\right\rangle=\frac{1}{\sqrt{2}}\left(\left|0 \lambda_{1}\right\rangle-\left|\lambda_{1} 0\right\rangle\right)$ to Charlie. As shown in Ref. [26], a phase error happens if Alice and Bob send $\left|\chi_{0}\right\rangle$ to Charlie and Charlie announces that the right detector clicks or Alice and Bob send $\left|\chi_{1}\right\rangle$ to Charlie and Charlie announces that the left detector clicks.

Denote $T_{0}^{R}$ as the probability that Charlie announces that the right detectors clicks while Alice and Bob send $\left|\chi_{0}\right\rangle$ to Charlie. Denote $T_{1}^{L}$ as the probability that Charlie announces that the left detectors clicks while Alice and Bob send $\left|\chi_{1}\right\rangle$ to Charlie. Denote $s_{1}$ as the yield of $\frac{1}{2}\left(\left|0 \lambda_{1}\right\rangle\left\langle 0 \lambda_{1}|+| \lambda_{1} 0\right\rangle\left\langle\lambda_{1} 0\right|\right)$. We can calculate the phase-flip error rate by [26]

$$
e^{\mathrm{ph}}=\frac{T_{0}^{R}+T_{1}^{L}}{2 s_{1}} .
$$

In the $Z$ windows of the real protocol, if Alice and Bob take the probability $\epsilon$ to send out a pulse of state $\rho_{z}$ and the probability $1-\epsilon$ to send out a pulse of state $|0\rangle\langle 0|$, then we can write the density matrix of the pulse pair in the $Z$ windows as

$$
\begin{aligned}
\rho_{r} & =\epsilon(1-\epsilon)\left(|0\rangle\left\langle 0\left|\otimes \rho_{z}+\rho_{z} \otimes\right| 0\right\rangle\langle 0|\right)+(1-\epsilon)^{2}|00\rangle\langle 00|+\epsilon^{2} \rho_{z} \otimes \rho_{z} \\
& =2 \epsilon(1-\epsilon) P_{1}\left(\mu_{z}\right)\left[\frac{1}{2}\left(\left|0 \lambda_{1}\right\rangle\left\langle 0 \lambda_{1}|+| \lambda_{1} 0\right\rangle\left\langle\lambda_{1} 0\right|\right)\right]+\left[1-2 \epsilon(1-\epsilon) P_{1}\left(\mu_{z}\right)\right] \rho_{c}
\end{aligned}
$$

where

$$
\rho_{c}=\frac{1}{1-2 \epsilon(1-\epsilon) P_{1}\left(\mu_{z}\right)}\left[(1-\epsilon)^{2}|00\rangle\langle 00|+\epsilon^{2} \rho_{z} \otimes \rho_{z}+\epsilon(1-\epsilon) \sum_{j=0, j \neq 1}^{N-1} P_{j}\left(\mu_{z}\right)\left(\left|0 \lambda_{j}\right\rangle\left\langle 0 \lambda_{j}|+| \lambda_{j} 0\right\rangle\left\langle\lambda_{j} 0\right|\right)\right] .
$$


Equation (12) clearly shows that the pulse pairs in the $Z$ windows are the classical mixture of the states $\rho_{1}=$ $\frac{1}{2}\left(\left|0 \lambda_{1}\right\rangle\left\langle 0 \lambda_{1}|+| \lambda_{1} 0\right\rangle\left\langle\lambda_{1} 0\right|\right)$ and $\rho_{c}$. We define a $Z$ window in which Alice and Bob send out a pulse pair of state $\rho_{1}$ as a $Z_{1}$ window. According to the discussion above and the tagged model [56], we can define the corresponding bits of the $Z_{1}$ windows as untagged bits whose maximum leakage information can be measured by $H\left(e^{\mathrm{ph}}\right)$, where $H(x)=-x \log _{2}(x)-$ $(1-x) \log _{2}(1-x)$ is the Shannon entropy. We define the rest of the bits of the $Z$ windows as tagged bits, whose bit values are assumed to be known by the eavesdropper. Finally, according to the famous Gottesman-Lo-Lütkenhaus-Preskill (GLLP) formula [56], we can get the secure final key rate by

$$
R=2 \epsilon(1-\epsilon) P_{1}\left(\mu_{z}\right) s_{1}\left[1-H\left(e^{\mathrm{ph}}\right)\right]-S_{z} f H(E),
$$

where $S_{z}$ is the yield of the events in the $Z$ windows, $f$ is the error correction efficiency factor, and $E$ is the bit error rate of the sifted keys.

\section{The decoy-state method}

To clearly show how to apply the decoy-state method to this protocol, we denote Alice's sources $|0\rangle,\left|e^{2 m \pi i / N} \sqrt{\mu_{x}}\right\rangle$, and $\left|e^{2 m^{\prime} \pi i / N} \sqrt{\mu_{y}}\right\rangle$ by $o_{A}, x_{A}$, and $y_{A}$, respectively, and we also denote Bob's sources $|0\rangle,\left|e^{2 n \pi i / N} \sqrt{\mu_{x}}\right\rangle$, and $\left|e^{2 n^{\prime} \pi i / N} \sqrt{\mu_{y}}\right\rangle$ by $o_{B}, x_{B}$, and $y_{B}$. We denote the source of pulse pairs by $\kappa_{A} \varsigma_{B}$, where $\kappa, \varsigma=o, x, y$. For simplicity, we omit the subscripts. For example, source $o x$ represents that Alice uses the source $o_{A}$ and Bob uses the source $x_{B}$. Without phase postselection, the density matrices of sources $x_{A}, y_{A}, x_{B}, y_{B}$ have a similar form to Eq. (3). Specifically, we have

$$
\rho_{w}=\sum_{j=0}^{N-1} P_{j}\left(\mu_{w}\right)\left|\lambda_{j}^{w}\right\rangle\left\langle\lambda_{j}^{w}\right| \quad(w=x, y)
$$

where

$$
\left|\lambda_{j}^{w}\right\rangle=\frac{1}{\sqrt{P_{j}\left(\mu_{w}\right)}} \sum_{k=0}^{\infty} \frac{\left(\sqrt{\mu_{w}}\right)^{k N+j}}{\sqrt{(k N+j) !}}|k N+j\rangle
$$

and $P_{j}\left(\mu_{w}\right)$ is defined in Eq. (5). Here, $\rho_{x}$ is the density matrix of sources $x_{A}$ and $x_{B}$, and $\rho_{y}$ is the density matrix of sources $y_{A}$ and $y_{B}$.

Denote the yield of sources $\kappa \varsigma$ by $S_{\kappa \zeta}$. Denote the yield of states $\left|0 \lambda_{j}^{w}\right\rangle\left\langle 0 \lambda_{j}^{w}\right|$ and $\left|\lambda_{j}^{w} 0\right\rangle\left\langle\lambda_{j}^{w} 0\right|$ by $Y_{v j}^{w}$ and $Y_{j v}^{w}$. In the original SNS protocol [26], as the continuously modulated phase-randomized WCS sources are used, we have

$$
Y_{v j}^{x}=Y_{v j}^{y}, \quad Y_{j v}^{x}=Y_{j v}^{y},
$$

but this equality no longer holds in this protocol. According to the properties of trace distance [46], we have

$$
\left|Y_{v j}^{x}-Y_{v j}^{y}\right| \leqslant \sqrt{1-\left(F_{x y}^{j}\right)^{2}}, \quad\left|Y_{j v}^{x}-Y_{j v}^{y}\right| \leqslant \sqrt{1-\left(F_{x y}^{j}\right)^{2}},
$$

where

$$
F_{x y}^{j}=\frac{\left|\left\langle\lambda_{j}^{x} \mid \lambda_{j}^{y}\right\rangle\right|}{\sqrt{\left.\left\langle\lambda_{j}^{x}\right| \lambda_{j}^{x}\right)\left\langle\lambda_{j}^{y} \mid \lambda_{j}^{y}\right\rangle}}=\frac{\sum_{k=0}^{\infty} \frac{\left(\mu_{x} \mu_{y}\right)^{(k N+j) / 2}}{(k N+j) !}}{\sqrt{\sum_{k=0}^{\infty} \frac{\mu_{x}^{k N+j}}{(k N+j) !} \sum_{k=0}^{\infty} \frac{\mu_{y}^{k N+j}}{(k N+j) !}}}
$$

is the fidelity of states $\left|\lambda_{j}^{x}\right\rangle$ and $\left|\lambda_{j}^{y}\right\rangle$.

Denote the yield of states $\left|0 \lambda_{1}\right\rangle$ and $\left|\lambda_{1} 0\right\rangle$ by $s_{01}$ and $s_{10}$ respectively. We have $s_{1}=\frac{1}{2}\left(s_{01}+s_{10}\right)$. We can get the lower bound of $s_{1}$ by either analytical formula or linear programming.

By combining the equations

$$
\begin{aligned}
S_{o x} & =\sum_{j=0}^{N-1} P_{j}\left(\mu_{x}\right) Y_{v j}^{x}=\sum_{j=0}^{N-1} P_{j}\left(\mu_{x}\right) Y_{v j}^{y}+\Delta, \\
S_{o y} & =\sum_{j=0}^{N-1} P_{j}\left(\mu_{y}\right) Y_{v j}^{y},
\end{aligned}
$$

where

$$
\Delta=\sum_{j=0}^{N-1} P_{j}\left(\mu_{x}\right)\left(Y_{v j}^{x}-Y_{v j}^{y}\right)
$$

we then have

$$
Y_{v 1}^{y}=\frac{P_{2}\left(\mu_{y}\right) S_{o x}-P_{2}\left(\mu_{x}\right) S_{o y}-\left[P_{0}\left(\mu_{x}\right) P_{2}\left(\mu_{y}\right)-P_{0}\left(\mu_{y}\right) P_{2}\left(\mu_{x}\right)\right] Y_{v 0}^{y}-P_{2}\left(\mu_{y}\right) \Delta-\xi}{P_{1}\left(\mu_{x}\right) P_{2}\left(\mu_{y}\right)-P_{1}\left(\mu_{y}\right) P_{2}\left(\mu_{x}\right)},
$$

where

$$
\xi=\sum_{j=3}^{N-1}\left[P_{j}\left(\mu_{x}\right) P_{2}\left(\mu_{y}\right)-P_{j}\left(\mu_{y}\right) P_{2}\left(\mu_{x}\right)\right] Y_{v j}^{y} .
$$

It is easy to check that $\xi \leqslant 0$ if the following condition holds:

$$
\frac{P_{1}\left(\mu_{x}\right)}{P_{1}\left(\mu_{y}\right)} \geqslant \frac{P_{2}\left(\mu_{x}\right)}{P_{2}\left(\mu_{y}\right)} \geqslant \frac{P_{j}\left(\mu_{x}\right)}{P_{j}\left(\mu_{y}\right)}, \quad j=3,4, \ldots, N-1,
$$

which can be easily examined given values of $\mu_{x}$ and $\mu_{y}$. In our numerical simulation, we found that Eq. (24) always holds. With

$$
Y_{v 0}^{y} \leqslant S_{o o}+\sqrt{1-F_{0}^{2}}, \quad \Delta \leqslant \Delta^{U}=\sum_{j=0}^{N-1} P_{j}\left(\mu_{x}\right) \sqrt{1-\left(F_{x y}^{j}\right)^{2}}, \quad s_{01} \geqslant Y_{v 1}^{y}-\sqrt{1-F_{1}^{2}},
$$


where

$$
F_{0}=\frac{1}{\sqrt{\sum_{k=0}^{\infty} \frac{\mu_{y}^{k N}}{(k N) !}}}, \quad F_{1}=\frac{\sum_{k=0}^{\infty} \frac{\left(\mu_{y} \mu_{z}\right)^{(k N+1) / 2}}{(k N+1) !}}{\sqrt{\sum_{k=0}^{\infty} \frac{\mu_{y}^{k N+1}(k N+1) !}{(k N)} \sum_{k=0}^{\infty} \frac{\mu_{y}^{k N+1}}{(k N+1) !}}},
$$

we have

$$
s_{01} \geqslant s_{01}^{L}=\frac{P_{2}\left(\mu_{y}\right) S_{o x}-P_{2}\left(\mu_{x}\right) S_{o y}-\left[P_{0}\left(\mu_{x}\right) P_{2}\left(\mu_{y}\right)-P_{0}\left(\mu_{y}\right) P_{2}\left(\mu_{x}\right)\right]\left(S_{o o}+\sqrt{1-F_{0}^{2}}\right)-P_{2}\left(\mu_{y}\right) \Delta^{U}}{P_{1}\left(\mu_{x}\right) P_{2}\left(\mu_{y}\right)-P_{1}\left(\mu_{y}\right) P_{2}\left(\mu_{x}\right)}-\sqrt{1-F_{1}^{2}} .
$$

By a similar method, we can prove that

$$
s_{10} \geqslant s_{10}^{L}=\frac{P_{2}\left(\mu_{y}\right) S_{x o}-P_{2}\left(\mu_{x}\right) S_{y o}-\left[P_{0}\left(\mu_{x}\right) P_{2}\left(\mu_{y}\right)-P_{0}\left(\mu_{y}\right) P_{2}\left(\mu_{x}\right)\right]\left(S_{o o}+\sqrt{1-F_{0}^{2}}\right)-P_{2}\left(\mu_{y}\right) \Delta^{U}}{P_{1}\left(\mu_{x}\right) P_{2}\left(\mu_{y}\right)-P_{1}\left(\mu_{y}\right) P_{2}\left(\mu_{x}\right)}-\sqrt{1-F_{1}^{2}},
$$

if Eq. (24) holds. Finally, we have $s_{1} \geqslant \frac{1}{2}\left(s_{01}^{L}+s_{10}^{L}\right)$.

The remaining task is estimating the upper bound of the phase-flip error rate $e^{\mathrm{ph}}$, which is equivalent to estimating the upper bounds of $T_{0}^{R}$ and $T_{1}^{L}$.

Denote $T_{+}^{R}$ as the probability that Charlie announces that the right detector clicks while Alice and Bob send out the pulse pairs in the $X_{1}$ window and their phases satisfy $m=n$. Denote $T_{-}^{L}$ as the probability that Charlie announces that the left detector clicks while Alice and Bob send out the pulse pairs in the $X_{1}$ window and their phases satisfy $m=$ $(n+N / 2) \bmod N$. Per the discussion above, we know that the pulse pairs in the $X_{1}$ window whose phases satisfy $m=n$ are the classical mixture of the state $\left|\varphi_{j}^{0}\right\rangle$. Thus, by denoting $t_{j}^{R}$ as the probability that Charlie announces that the right detector clicks while Alice and Bob send out the pulse pairs of state $\left|\varphi_{j}^{0}\right\rangle$, we have

$$
T_{+}^{R}=\sum_{j=0}^{N-1} P X_{j}\left(\mu_{x}\right) t_{j}^{R}
$$

Denote $T_{00}$ and $T_{00}^{\prime}$ as the probability that Charlie announces that the right detector clicks and the left detector clicks while Alice and Bob send out a vacuum pulse pair. According to the properties of trace distance, we have

$$
\left|t_{0}^{R}-T_{00}\right| \leqslant \sqrt{1-F_{00}^{2}}, \quad\left|t_{1}^{R}-T_{0}^{R}\right| \leqslant \sqrt{1-\left[F_{11}^{+}(0)\right]^{2}},
$$

where

$$
F_{00}=\frac{\left|\left\langle\varphi_{0}^{0} \mid 0\right\rangle\right|}{\sqrt{\left\langle\varphi_{0}^{0} \mid \varphi_{0}^{0}\right\rangle\langle 0 \mid 0\rangle}}=\frac{1}{\sqrt{\sum_{k=0}^{\infty} \frac{\left(2 \mu_{x}\right)^{k N}}{(k N) !}}}
$$

and

$$
\begin{aligned}
F_{11}^{+}(q) & =\frac{\left|\left\langle\varphi_{1}^{q} \mid \chi_{0}\right\rangle\right|}{\sqrt{\left\langle\varphi_{1}^{q} \mid \varphi_{1}^{q}\right\rangle\left\langle\chi_{0} \mid \chi_{0}\right\rangle}}=\frac{\sqrt{\operatorname{Re}_{+}^{2}+\operatorname{Im}^{2}}}{\sqrt{\sum_{k=0}^{\infty} \frac{\left(2 \mu_{x}\right)^{k N+1}}{(k N+1) !} \sum_{k=0}^{\infty} \frac{\mu_{z}^{k N+1}}{(k N+1) !}}} \\
\operatorname{Re}_{+} & =\frac{1}{\sqrt{2}} \sum_{k=0}^{\infty} \frac{\left(\mu_{x} \mu_{z}\right)^{(k N+1) / 2}}{(k N+1) !}\left[1+\cos \frac{2 \pi}{N} q(k N+1)\right], \\
\operatorname{Im} & =\frac{1}{\sqrt{2}} \sum_{k=0}^{\infty} \frac{\left(\mu_{x} \mu_{z}\right)^{(k N+1) / 2}}{(k N+1) !} \sin \frac{2 \pi}{N} q(k N+1) .
\end{aligned}
$$

Combining Eqs. (28) and (29), we have

$$
\begin{aligned}
T_{0}^{R} \leqslant T_{0}^{R, U}= & \frac{T_{+}^{R}-P X_{0}\left(\mu_{x}\right)\left(T_{00}-\sqrt{1-F_{00}^{2}}\right)}{P X_{1}\left(\mu_{x}\right)} \\
& +\sqrt{1-\left[F_{11}^{+}(0)\right]^{2}} .
\end{aligned}
$$

With a similar method, we have

$$
\begin{aligned}
T_{1}^{L} \leqslant T_{1}^{L, U}= & \frac{T_{-}^{L}-P X_{0}\left(\mu_{x}\right)\left(T_{00}^{\prime}-\sqrt{1-F_{00}^{2}}\right)}{P X_{1}\left(\mu_{x}\right)} \\
& +\sqrt{1-\left[F_{11}^{-}\left(\frac{N}{2}\right)\right]^{2}},
\end{aligned}
$$

where

$$
\begin{aligned}
& F_{11}^{-}(q)=\frac{\left|\left\langle\varphi_{1}^{q} \mid \chi_{1}\right\rangle\right|}{\sqrt{\left\langle\varphi_{1}^{q} \mid \varphi_{1}^{q}\right\rangle\left\langle\chi_{1} \mid \chi_{1}\right\rangle}}=\frac{\sqrt{\operatorname{Re}_{-}^{2}+\operatorname{Im}^{2}}}{\sqrt{\sum_{k=0}^{\infty} \frac{\left(2 \mu_{x}\right)^{k N+1}}{(k N+1) !} \sum_{k=0}^{\infty} \frac{\mu_{z}^{k N+1}}{(k N+1) !}}}, \\
& \operatorname{Re}_{-}=\frac{1}{\sqrt{2}} \sum_{k=0}^{\infty} \frac{\left(\mu_{x} \mu_{z}\right)^{(k N+1) / 2}}{(k N+1) !}\left[1-\cos \frac{2 \pi}{N} q(k N+1)\right],
\end{aligned}
$$

and Im has already been shown in Eq. (32). Finally, we have

$$
e^{\mathrm{ph}} \leqslant \frac{T_{0}^{R, U}+T_{1}^{L, U}}{s_{01}^{L}+s_{10}^{L}}
$$

With all these formulas, we can now calculate the secure final key rate according to the observed values in the experiment.

We have obtained the explicit formulas above for key rate calculation. In our calculation below, we shall use our analytic formulas above. Definitely, the key rate here can also be 
calculated through linear programming. It is

$$
\begin{aligned}
\text { Minimize } \mathrm{s}_{1} & =\frac{1}{2}\left(s_{01}+s_{10}\right) \\
\text { subject to } S_{o x} & =\sum_{j=0}^{N-1} P_{j}\left(\mu_{x}\right) Y_{v j}^{x}, \quad S_{o y}=\sum_{j=0}^{N-1} P_{j}\left(\mu_{y}\right) Y_{v j}^{y}, \\
S_{x o} & =\sum_{j=0}^{N-1} P_{j}\left(\mu_{x}\right) Y_{j v}^{x}, \quad S_{y o}=\sum_{j=0}^{N-1} P_{j}\left(\mu_{y}\right) Y_{j v}^{y}, \\
\left|S_{o o}-Y_{0 v}^{y}\right| & \leqslant \sqrt{1-F_{0}^{2}}, \quad\left|S_{o o}-Y_{v 0}^{y}\right| \leqslant \sqrt{1-F_{0}^{2}}, \\
\left|Y_{v j}^{x}-Y_{v j}^{y}\right| & \leqslant \sqrt{1-\left(F_{x y}^{j}\right)^{2}}, \quad\left|Y_{j v}^{x}-Y_{j v}^{y}\right| \leqslant \sqrt{1-\left(F_{x y}^{j}\right)^{2}}, \\
\left|s_{01}-Y_{v 1}^{y}\right| & \leqslant \sqrt{1-F_{1}^{2}}, \quad\left|s_{10}-Y_{1 v}^{y}\right| \leqslant \sqrt{1-F_{1}^{2}} .
\end{aligned}
$$

After we get the lower bound of $s_{1}$ through linear programming, the remaining process is the same as the above method.

\section{THE NUMERICAL SIMULATION}

In this section, we shall show some numerical simulation results.

We use the linear model to simulate the observed values [31,34]. The distance between Alice and Charlie and the distance between Bob and Charlie is assumed to be the same. The properties of the two detectors of Charlie are assumed to be the same. The lowest intensities of the sources in the decoy window are set as $\mu_{x} \geqslant 0.001$ and $\mu_{y} \geqslant 0.002$. Then $\mu_{x}, \mu_{y}$ and the remaining parameters including $\mu_{z}$ and $\epsilon$ are optimized to obtained the highest key rates. In principle, the values of $\mu_{x}, \mu_{y}$ should be optimized in the interval $[0,+\infty)$, but limited by the laboratory technique, it is pretty hard to attain stable sources with intensities below 0.001. The "distance" shown in the figures in this section means the length of fiber between Alice and Bob. The experimental parameters used in our numerical simulation are listed in Table I.

Figure 1 shows the key rate of the SNS protocol with different numbers of phase values. The dashed cyan curve is the PLOB bound, which was established by Pirandola, Laurenza, Ottaviani, and Banchi to measure the linear upper bound of the key rate of QKD [24]. The key rates almost coincide for the cases $N>12$. Thus the curves for $N>12$ are not listed in this figure. Figure 1 shows that with only six phase values, the key rate of the SNS protocol can exceed the PLOB bound and, with 12 phase values, the key rate is

TABLE I. List of experimental parameters used in the numerical simulations. Here, $p_{d}$ is the dark count rate of Charlie's detectors, $e_{d}$ is the misalignment-error probability, $\eta_{d}$ is the detection efficiency of Charlie's detectors, $f$ is the error correction inefficiency, and $\alpha_{f}$ is the fiber loss coefficient.

\begin{tabular}{lcccc}
\hline \hline$p_{d}$ & $e_{d}(\%)$ & $\eta_{d}(\%)$ & $f$ & $\alpha_{f}(\mathrm{~dB} / \mathrm{km})$ \\
\hline $1.0 \times 10^{-8}$ & 3 & 30.0 & 1.1 & 0.2 \\
\hline \hline
\end{tabular}

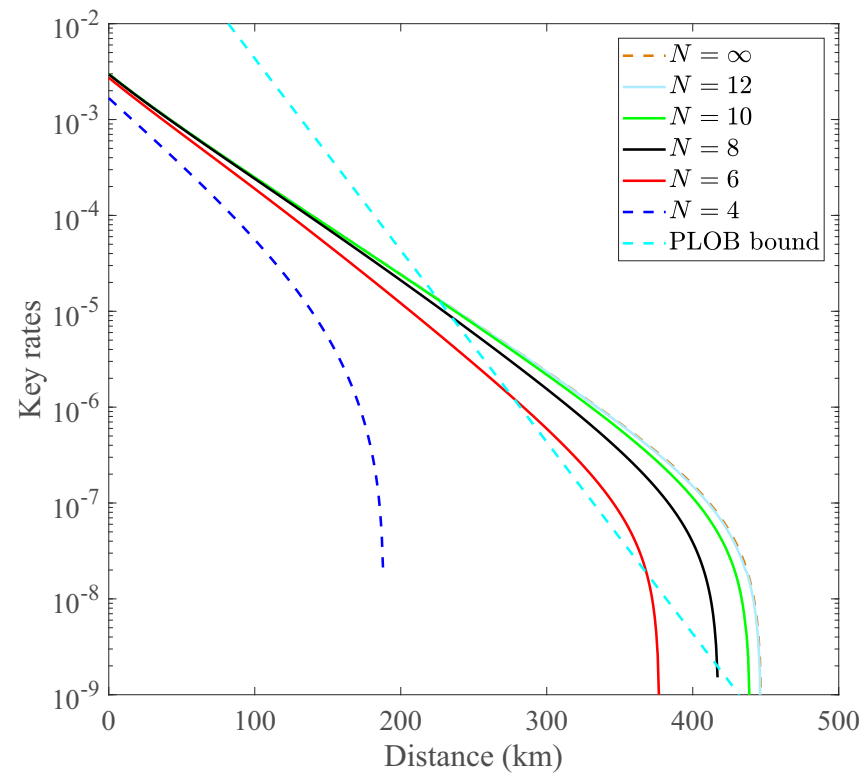

FIG. 1. The key rates of the SNS protocol with different numbers of phase values. The experimental parameters used in the numerical simulation are shown in Table I. The PLOB bound is used to measure the linear upper bound of the key rate of QKD [24].

very close to the SNS protocol with continuously modulated phase-randomized WCS sources.

Figure 2 shows the key rate of the SNS protocol with AOPP [35] and different numbers of phase values, while Fig. 3 shows a comparison of the key rates of the original SNS protocol and the AOPP method. The AOPP method is an error rejection process through two-way classical communication, which is

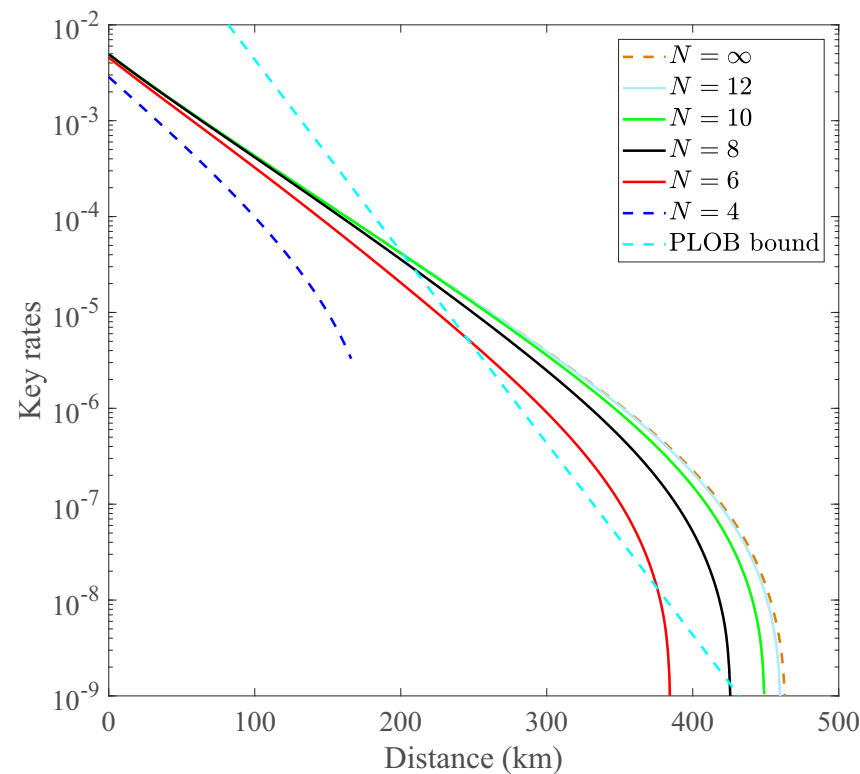

FIG. 2. The key rates of the SNS protocol with AOPP [35] and different numbers of phase values. The experimental parameters used in the numerical simulation are shown in Table I. The PLOB bound is used to measure the linear upper bound of the key rate of QKD [24]. 


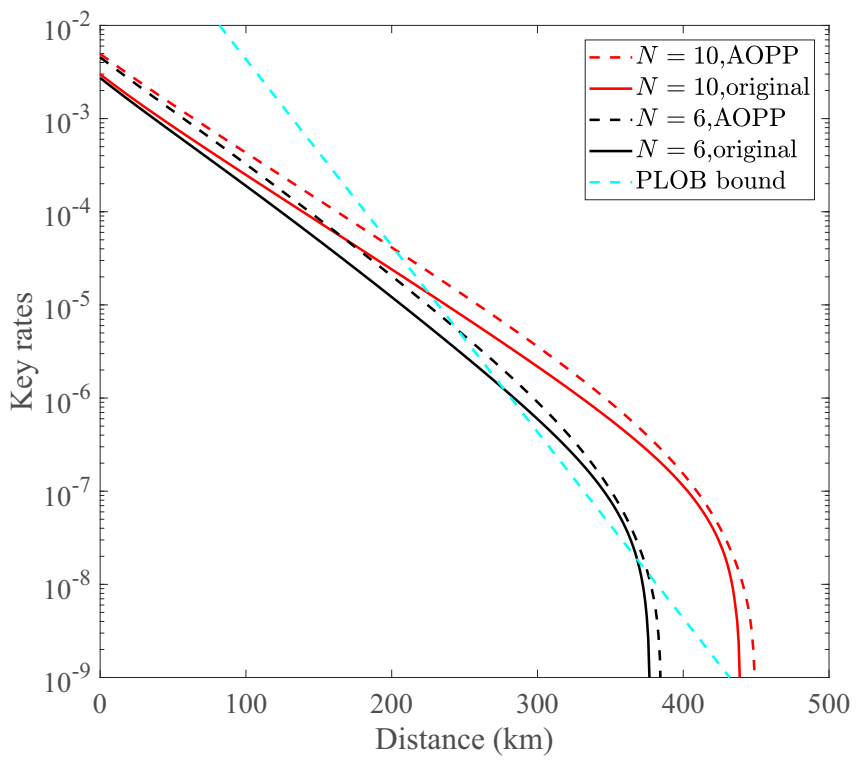

FIG. 3. Comparison of the key rates of the original SNS protocol and the AOPP method [35]. The experimental parameters used in the numerical simulation are shown in Table I. The PLOB bound is used to measure the linear upper bound of the key rate of QKD [24].

only related to the sifted keys. As the property of no bit-flip error in the untagged bits holds in the SNS protocol with discrete-phase-randomized WCS sources, we can directly apply the formulas derived in our previous work [35] to calculate the key rate after AOPP. Figure 3 shows that key rates of the SNS protocol with AOPP obviously exceed those of the original SNS protocol. While the distance between Alice and Bob is less than $150 \mathrm{~km}$, the key rates of the AOPP method with six phase values are even higher than those of the original SNS protocol with 10 phase values.

Figure 4 is a comparison of the key rates of the 4-intensity protocol and the 3-intensity protocol. In the 4-intensity protocol introduced in Sec. II A, $\mu_{y}$ does not have to be equal to $\mu_{z}$. By adding a constraint that $\mu_{y}=\mu_{z}$, we get the 3-intensity protocol [31], which is a more convenient protocol in the experiments. Figure 4 shows that while the number of phase values is large, which is closer to the case of a continuously modulated protocol, the key rates of the 3-intensity protocol are almost the same as those of the 4-intensity protocol. As the number of phase values decreases, the key rate gap between the two protocols gradually increases.

\section{CONCLUSION}

In this paper, we study the SNS protocol with discretephase-randomized WCS sources. Starting from the security

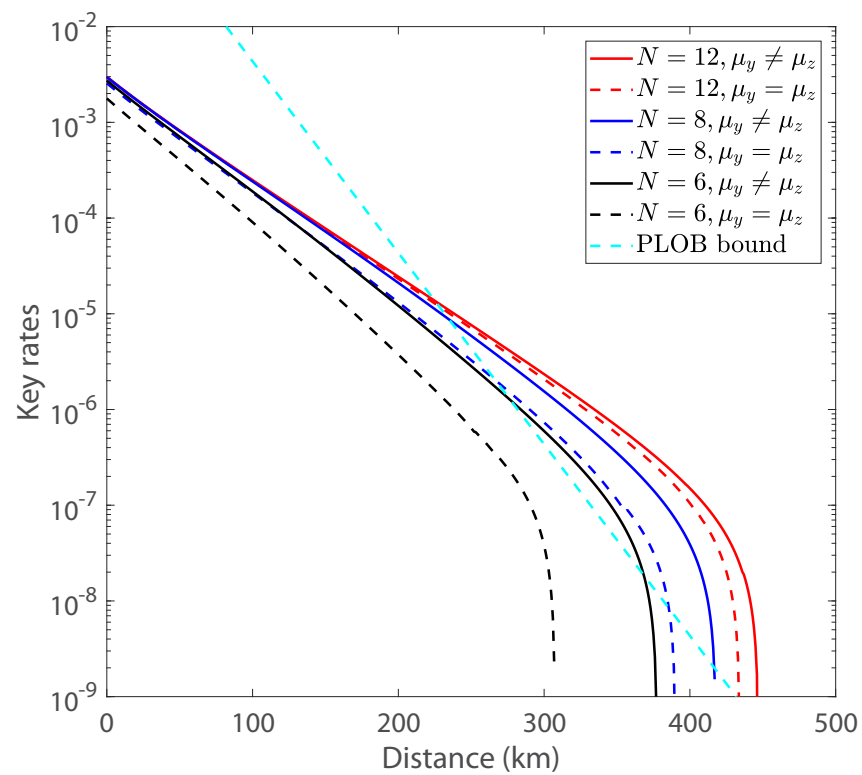

FIG. 4. Comparison of the key rates of the 4-intensity protocol $\left(\mu_{y} \neq \mu_{z}\right)$ and the 3 -intensity protocol $\left(\mu_{y}=\mu_{z}\right)$. The experimental parameters used in the numerical simulation are shown in Table I. The PLOB bound is used to measure the linear upper bound of the key rate of QKD [24].

proof, we get the formula of the phase-flip error rate when discrete-phase-randomized coherent-state sources are used. With our derivations, we also get the lower bound of the yield of untagged bits. The lower bound can be calculated by either analytical formula or linear programming. Our numerical simulation results show that with only six phase values, the key rates of the SNS protocol can exceed the PLOB bound and, with 12 phase values, the key rates are very close to the SNS protocol with continuously modulated phase-randomized WCS sources. The AOPP method proposed in Ref. [35] can be directly applied here, and the numerical results show that the advantage of the AOPP method still holds in the SNS protocol with discrete-phase-randomized WCS sources.

\section{ACKNOWLEDGMENTS}

We acknowledge financial support in part by Ministry of Science and Technology of China through National Key Research and Development Program of China Grant No. 2017YFA0303901, National Natural Science Foundation of China Grants No. 11474182, 11774198, 11974204, U1738142, and 61801126, and Natural Science Foundation of Shandong Province Grant No. ZR2019LLZ004.
[1] C. H. Bennett and G. Brassard, Quantum cryptography: Public key distribution and coin tossing, in Proceedings of the IEEE International Conference on Computers, Systems, and Signal Processing (IEEE, Piscataway, NJ, 1984) pp. 175-179.
[2] A. K. Ekert, Quantum Cryptography Based on Bell's Theorem, Phys. Rev. Lett. 67, 661 (1991)

[3] S. Pirandola, U. Andersen, L. Banchi, M. Berta, D. Bunandar, R. Colbeck, D. Englund, T. Gehring, C. Lupo, C. Ottaviani, 
J. Pereira, M. Razavi, J. S. Shaari, M. Tomamichel, V. C. Usenko, G. Vallone, P. Villoresi, and P. Wallden, Advances in quantum cryptography, arXiv:1906.01645.

[4] F. Xu, X. Ma, Q. Zhang, H.-K. Lo, and J.-W. Pan, Secure quantum key distribution with realistic devices, Rev. Mod. Phys. 92, 025002 (2020).

[5] N. Gisin, G. Ribordy, W. Tittel, and H. Zbinden, Quantum cryptography, Rev. Mod. Phys. 74, 145 (2002).

[6] N. Gisin and R. Thew, Quantum communication, Nat. Photonics 1, 165 (2007).

[7] V. Scarani, H. Bechmann-Pasquinucci, N. J. Cerf, M. Dušek, N. Lütkenhaus, and M. Peev, The security of practical quantum key distribution, Rev. Mod. Phys. 81, 1301 (2009).

[8] P. W. Shor and J. Preskill, Simple Proof of Security of the BB84 Quantum Key Distribution Protocol, Phys. Rev. Lett. 85, 441 (2000).

[9] M. Koashi, Simple security proof of quantum key distribution based on complementarity, New J. Phys. 11, 045018 (2009).

[10] W.-Y. Hwang, Quantum Key Distribution with High Loss: Toward Global Secure Communication, Phys. Rev. Lett. 91, 057901 (2003).

[11] X.-B. Wang, Beating the Photon-Number-Splitting Attack in Practical Quantum Cryptography, Phys. Rev. Lett. 94, 230503 (2005).

[12] H.-K. Lo, X. Ma, and K. Chen, Decoy State Quantum Key Distribution, Phys. Rev. Lett. 94, 230504 (2005).

[13] X.-B. Wang, X.-L. Hu, and Z.-W. Yu, Practical Long-Distance Side-Channel-Free Quantum Key Distribution, Phys. Rev. Appl. 12, 054034 (2019).

[14] B. Kraus, N. Gisin, and R. Renner, Lower and Upper Bounds on the Secret-Key Rate for Quantum Key Distribution Protocols Using One-Way Classical Communication, Phys. Rev. Lett. 95, 080501 (2005).

[15] S. L. Braunstein and S. Pirandola, Side-Channel-Free Quantum Key Distribution, Phys. Rev. Lett. 108, 130502 (2012).

[16] H.-K. Lo, M. Curty, and B. Qi, Measurement-DeviceIndependent Quantum Key Distribution, Phys. Rev. Lett. 108, 130503 (2012).

[17] H.-L. Yin, T.-Y. Chen, Z.-W. Yu, H. Liu, L.-X. You, Y.-H. Zhou, S.-J. Chen, Y. Mao, M.-Q. Huang, W.-J. Zhang, H. Chen, M. J. Li, D. Nolan, F. Zhou, X. Jiang, Z. Wang, Q. Zhang, X.-B. Wang, and J.-W. Pan, Measurement-Device-Independent Quantum Key Distribution over a 404 km Optical Fiber, Phys. Rev. Lett. 117, 190501 (2016).

[18] S.-K. Liao, W.-Q. Cai, W.-Y. Liu, L. Zhang, Y. Li, J.-G. Ren, J. Yin, Q. Shen, Y. Cao, Z.-P. Li, F.-Z. Li, X.-W. Chen, L.-H. Sun, J.-J. Jia, J.-C. Wu, X.-J. Jiang, J.-F. Wang, Y.-M. Huang, Q. Wang, Y.-L. Zhou et al., Satellite-to-ground quantum key distribution, Nature (London) 549, 43 (2017).

[19] A. Boaron, G. Boso, D. Rusca, C. Vulliez, C. Autebert, M. Caloz, M. Perrenoud, G. Gras, F. Bussières, M.-J. Li, D. Nolan, A. Martin, and H. Zbinden, Secure Quantum Key Distribution over 421 km of Optical Fiber, Phys. Rev. Lett. 121, 190502 (2018).

[20] J.-P. Chen, C. Zhang, Y. Liu, C. Jiang, W. Zhang, X.-L. Hu, J.-Y. Guan, Z.-W. Yu, H. Xu, J. Lin, M.-J. Li, H. Chen, H. Li, L. You, Z. Wang, X.-B. Wang, Q. Zhang, and J.-W. Pan, Sendingor-Not-Sending with Independent Lasers: Secure Twin-Field Quantum Key Distribution over 509 km, Phys. Rev. Lett. 124, 070501 (2020).
[21] J. Yin, Y.-H. Li, S.-K. Liao, M. Yang, Y. Cao, L. Zhang, J.-G. Ren, W.-Q. Cai, W.-Y. Liu, S.-L. Li, R. Shu, Y.-M. Huang, L. Deng, L. Li, Q. Zhang, N.-L. Liu, Y.-A. Chen, C.-Y. Lu, X.-B. Wang, F. Xu et al., Entanglement-based secure quantum cryptography over 1,120 kilometres, Nature (London) 582, 501 (2020).

[22] S. Pirandola, R. García-Patrón, S. L. Braunstein, and S. Lloyd, Direct and Reverse Secret-Key Capacities of a Quantum Channel, Phys. Rev. Lett. 102, 050503 (2009).

[23] M. Takeoka, S. Guha, and M. M. Wilde, Fundamental rate-loss tradeoff for optical quantum key distribution, Nat. Commun. 5, 5235 (2014).

[24] S. Pirandola, R. Laurenza, C. Ottaviani, and L. Banchi, Fundamental limits of repeaterless quantum communications, Nat. Commun. 8, 15043 (2017).

[25] M. Lucamarini, Z. L. Yuan, J. F. Dynes, and A. J. Shields, Overcoming the rate-distance limit of quantum key distribution without quantum repeaters, Nature (London) 557, 400 (2018).

[26] X.-B. Wang, Z.-W. Yu, and X.-L. Hu, Twin-field quantum key distribution with large misalignment error, Phys. Rev. A 98, 062323 (2018).

[27] K. Tamaki, H.-K. Lo, W. Wang, and M. Lucamarini, Information theoretic security of quantum key distribution overcoming the repeaterless secret key capacity bound, arXiv:1805.05511.

[28] J. Lin and N. Lütkenhaus, Simple security analysis of phasematching measurement-device-independent quantum key distribution, Phys. Rev. A 98, 042332 (2018).

[29] C. Cui, Z.-Q. Yin, R. Wang, W. Chen, S. Wang, G.-C. Guo, and Z.-F. Han, Twin-Field Quantum Key Distribution without Phase Postselection, Phys. Rev. Appl. 11, 034053 (2019).

[30] M. Curty, K. Azuma, and H. K. Lo, Simple security proof of twin-field type quantum key distribution protocol, npj Quantum Inf. 5, 64 (2019).

[31] Z. W. Yu, X. L. Hu, C. Jiang, H. Xu, and X.-B. Wang, Sendingor-not-sending twin-field quantum key distribution in practice, Sci. Rep. 9, 3080 (2019).

[32] K. Maeda, T. Sasaki, and M. Koashi, Repeaterless quantum key distribution with efficient finite-key analysis overcoming the rate-distance limit, Nat. Commun. 10, 3140 (2019).

[33] F. Y. Lu, Z. Q. Yin, R. Wang, G.-J. Fan-Yuan, S. Wang, D.-Y He, W. Chen, W. Huang, B.-J. Xu, G.-C. Guo, and Z.-F. Han, Practical issues of twin-field quantum key distribution, New J. Phys. 21, 123030 (2019).

[34] C. Jiang, Z. W. Yu, X. L. Hu, and X.-B. Wang, Unconditional Security of Sending or Not Sending Twin-Field Quantum Key Distribution with Finite Pulses, Phys. Rev. Appl. 12, 024061 (2019).

[35] H. Xu, Z. W. Yu, C. Jiang, X.-L. Hu, and X.-B. Wang, Sendingor-not-sending twin-field quantum key distribution: Breaking the direct transmission key rate, Phys. Rev. A 101, 042330 (2020).

[36] F. Grasselli, A. Navarrete, and M. Curty, Asymmetric twin-field quantum key distribution, New J. Phys. 21, 113032 (2019).

[37] W. Wang and H. K. Lo, Simple method for asymmetric twinfield quantum key distribution, New J. Phys. 22, 013020 (2020).

[38] X.-L. Hu, C. Jiang, Z.-W. Yu, and X.-B. Wang, Sending-ornot-sending twin-field protocol for quantum key distribution with asymmetric source parameters, Phys. Rev. A 100, 062337 (2019). 
[39] C.-H. Zhang, C.-M. Zhang, and Q. Wang, Twin-field quantum key distribution with modified coherent states, Opt. Lett. 44, 1468 (2019).

[40] X.-Y. Zhou, C.-H. Zhang, C.-M. Zhang, and Q. Wang, Asymmetric sending or not sending twin-field quantum key distribution in practice, Phys. Rev. A 99, 062316 (2019).

[41] M. Minder, M. Pittaluga, G. Roberts, M. Lucamarini, J. Dynes, Z. Yuan, and A. Shields, Experimental quantum key distribution beyond the repeaterless secret key capacity, Nat. Photonics 13, 334 (2019).

[42] Y. Liu, Z.-W. Yu, W. Zhang, J.-Y. Guan, J.-P. Chen, C. Zhang, X.-L. Hu, H. Li, C. Jiang, J. Lin, T.-Y. Chen, L. You, Z. Wang, X.-B. Wang, Q. Zhang, and J.-W. Pan, Experimental Twin-Field Quantum Key Distribution through Sending or Not Sending, Phys. Rev. Lett. 123, 100505 (2019).

[43] S. Wang, D.-Y. He, Z.-Q. Yin, F.-Y. Lu, C.-H. Cui, W. Chen, Z. Zhou, G.-C. Guo, and Z.-F. Han, Beating the Fundamental Rate-Distance Limit in a Proof-of-Principle Quantum Key Distribution System, Phys. Rev. X 9, 021046 (2019).

[44] X. Zhong, J. Hu, M. Curty, L. Qian, and H.-K. Lo, Proofof-Principle Experimental Demonstration of Twin-Field Type Quantum Key Distribution, Phys. Rev. Lett. 123, 100506 (2019).

[45] C. Jiang, X.-L. Hu, H. Xu, Z.-W. Yu, and X.-B. Wang, Zigzag approach to higher key rate of sending-or-not-sending twin field quantum key distribution with finite-key effects, New J. Phys. 22, 053048 (2020).

[46] Z. Cao, Z. Zhang, H.-K. Lo, and X. Ma, Discrete-phaserandomized coherent state source and its application in quantum key distribution, New J. Phys. 17, 053014 (2015).

[47] K. Tamaki, H.-K. Lo, C.-H. F. Fung, and B. Qi, Phase encoding schemes for measurement-device-independent quantum key distribution with basis-dependent flaw, Phys. Rev. A 85, 042307 (2012).
[48] X.-B. Wang, Three-intensity decoy-state method for deviceindependent quantum key distribution with basis-dependent errors, Phys. Rev. A 87, 012320 (2013).

[49] F. Xu, M. Curty, B. Qi, and H.-K. Lo, Practical aspects of measurement-device-independent quantum key distribution, New J. Phys. 15, 113007 (2013).

[50] M. Curty, F. Xu, W. Cui, C. C. W. Lim, K. Tamaki, and H.-K. Lo, Finite-key analysis for measurement-deviceindependent quantum key distribution, Nat. Commun. 5, 3732 (2014).

[51] F. Xu, H. Xu, and H.-K. Lo, Protocol choice and parameter optimization in decoy-state measurement-deviceindependent quantum key distribution, Phys. Rev. A 89, 052333 (2014).

[52] Y.-H. Zhou, Z.-W. Yu, and X.-B. Wang, Making the decoystate measurement-device-independent quantum key distribution practically useful, Phys. Rev. A 93, 042324 (2016).

[53] I. W. Primaatmaja, E. Lavie, K. T. Goh, C. Wang, and C. C. W. Lim, Versatile security analysis of measurementdevice-independent quantum key distribution, Phys. Rev. A 99, 062332 (2019).

[54] Z. Cao, Discrete-phase-randomized measurement-deviceindependent quantum key distribution, Phys. Rev. A 101, 062325 (2020).

[55] G. C. Lorenzo, L. Wooltorton, and M. Razavi, Twin-field quantum key distribution with fully discrete phase randomization, arXiv:2008.03222.

[56] D. Gottesman, H.-K. Lo, N. Lütkenhaus, and J. Preskill, Security of quantum key distribution with imperfect devices, in 2004 International Symposium on Information Theory (IEEE, Piscataway, NJ, 2004), p. 136.

[57] C.-M. Zhang, Y.-W. Xu, R. Wang, and Q. Wang, Twinfield quantum key distribution with discrete-phase-randomized sources, arXiv:2008.05277. 